\title{
Editorial note to: \\ Jerome Kristian and Rainer K. Sachs, Observations in cosmology
}

\author{
George Ellis
}

Published online: 18 November 2010

(C) Springer Science+Business Media, LLC 2010

Keywords Anisotropic, inhomogeneous cosmological models ·

Cosmological effects · Golden Oldie

\section{Introduction}

This paper is one of the least appreciated fundamental papers in theoretical cosmology. It carefully develops a direct observationally based approach to cosmology, in contrast to the standard model-fitting approach; and it does so in a comprehensive and systematic way. However it only does so in the form of a power-series solution (as was the custom at the time in cosmology): later papers have taken the approach further.

The basic issue is that there is an ongoing tension in cosmology between theory and observation. Theoretical models have given us major insights into the nature of processes taking place, but have of necessity utilized very simple geometrical models of spacetime and the matter distribution in it. Indeed the standard theory of cosmology is based on highly simplified models: the spatially homogeneous and isotropic Robertson-Walker (RW) family of spacetimes, distinguished from each other by the values of a few parameters to be observationally determined by astronomical observations. These basic models are then modified to perturbed RW models that statistically represent the kinds of inhomogeneities seen. The statistics of these models are fitted to the actual observations, and then used to constrain theories of structure formation and background evolution, hence putting further constraints on the background model

The republication of the original paper can be found in this issue following the editorial note and online via doi:10.1007/s10714-010-1114-1.

G. Ellis $(\bowtie)$

Mathematics Department, University of Cape Town, Rondebosch 7700, Cape Town, South Africa e-mail: George.Ellis@uct.ac.za 
parameters. The outcome is a perturbed background FLRW model, with a completely determined set of parameters selecting a specific best-fit model from the overall family of such models.

But these are statistical descriptions: they do not describe what is actually there. What is really there is often surprising and contrary to all present models- the expansion of the universe, large scale motions, walls, and huge voids are examples of observational discoveries that were resisted by the theoretical community of the time, and then eventually incorporated into more complex models (often in a patchwork way). So observations have continually shown that things are more complex than indicated by simple models, and have lead to new generations of more complex models. Even then, the resulting theories are unable to predict what actually exists: our specific Galaxy, Sun, planet Earth; they can only predict the statistics of the general kind of thing that will come into existence (galaxies, stars, planets). However we also want to know in detail what is actually there, as is made clear for example by projects such as the SDSS and $2 \mathrm{dF}$ galaxy surveys. Thus one can envisage a purely observationally based programme, where one simply determines what is out there without biassing it by imposing some pre-determined geometric model. This is relatively easy to do in the Newtonian case, but not so easy in the context of general relativity theory.

\section{The Kristian and Sachs paper}

The Kristian and Sachs paper carries out the project of showing how to determine the spacetime structure directly from astronomical observations in the generic General Relativity case, i.e without assuming any preconceived geometry for the universe, using a power series in distance from the origin of observation. This develops a line of earlier work carried out by McCrea [15] (which is however not listed in the references).

The paper has three parts: a generic analysis of observations in cosmology (with no symmetries assumed and without imposing any field equations), then the implications when the Einstein field equations for pressure-free matter are imposed, and finally a summary of the results obtained in a Cartesian-tensor formalism. It uses the $1+3$ covariant formalism for general relativistic fluids developed by the Hamburg Group of Engelbert Schücking, Jürgen Ehlers, Rainer Sachs, and Manfred Trümper, partly presented by Ehlers [6] in an earlier paper. Together with a paper by Hawking [10], this was the first generally available presentation of the full covariant formalism, including the Weyl tensor. It also uses the theory of ray bundles developed by the Hamburg group, which underlie geometric optics in a curved spacetime.

The paper gives power series expressions for observations in a general cosmological spacetime: redshift versus luminosity distance, number counts versus luminosity distance, and so on, relating them to the fluid kinematic quantities [6] and to the electric and magnetic parts of the Weyl tensor [7,10]. It has a number of important features. Firstly, it gives a clear statement of the relation between observed intensities, distance definitions, and redshifts - a subject that was in a state of confusion at the time. Secondly, it showed that area distance and angular diameter distance for null geodesics are equal to each other in a general spacetime. This fundamental result was proved by Kristian and Sachs in a power series form [equation (37) in this paper], but was 
then shown to be exact by Roger Penrose (see the note after that equation), and was later identified as the reciprocity theorem of Etherington [9] (a clear statement of the theorem and its observational implications is given in [7]). Thirdly, it gives the first discussion of how distortion effects due to the Weyl tensor could give cosmological information of significance. This has now grown into the burgeoning field of gravitational lensing studies, that provides crucial information for cosmology. Fourthly, it discusses the major missing element in cosmological observations: namely proper motions of cosmological objects (the linear version was given in [6]; here that result is extended to non-linear terms). Fifthly, it gives numerical estimates for the magnitudes of the various terms that characterize anisotropy in cosmology: some of these estimates have not been bettered even today.

Overall, it is a remarkable pioneering paper that provided impetus for other generic studies of cosmological observations, and to applications in restricted classes of models.

\section{Extension of domain}

A major extension of the Kristian and Sachs results was provided by [8] who extended the analysis to a set of exact results showing how to (in principle) determine the geometry of the past null cone from astronomical observations. This analysis usefully shows precisely what data is needed to determine the geometry of the past null cone, and then to integrate off the past null cone to the past. The analysis shows that it is not even in principle possible to predict to the future without a suitable 'no interference' condition, unless the universe is a spatially closed 'small universe' that we have already seen right round. This situation is completely obscured when we assume a FLRW geometry $\mathrm{ab}$ initio (thus changing the background field equations from hyperbolic to ODEs).

The method also makes very clear the growth of uncertainty with distance (i.e. with redshift) and the limitations of the domain where one can reliably establish spacetime geometry from observations, limited by the visual horizon occurring at the Last Scattering Surface (LSS). From this viewpoint, the data available from the Cosmic Microwave Background radiation temperature anisotropies can be used to place constraints on the LSS geometry.

The possibility of carrying out this observational cosmology programme is dependent on two conditions, apart from the necessity of determining the transverse motion of matter (through measuring proper motions in the sky). First, one needs reliable standard candles to act as distance indicators; nowadays these are provided by Type Ia supernovae. Second one needs to be able to estimate the density of matter at each point by observations of astronomical objects. This part of the project has been made far more difficult by the discovery of the dominance of local dynamics by dark matter. Nevertheless astrophysical estimates through observations of local dynamics, together with gravitational lensing, mean that the situation is not hopeless. Similar problems arise as regards a cosmological constant: one can carry out the geometric reconstruction programme for any value of the cosmological constant $\Lambda$. Its value has to be determined from geometrical assumptions (e.g. assuming a 
FLRW geometry) or from local physical effects (for example how it affects structure formation).

\section{Restricted examples: spherical symmetry}

Given that observations show the universe is almost spherically symmetric about us, one can ask if a restricted version of the project can be carried out for spherically symmetric spacetimes: can one determine the arbitrary functions in a spherically symmetric dust model from astronomical observations made by an observer near the centre? That this is possible in principle, as far as the basic cosmological observations (area distances and number counts) are concerned, is shown by a theorem by Mustapha et al. [17]. Details of how to carry this out in practice have been developed in $[11,13,14]$. This should be regarded as part of the general approach adopted by Kristian and Sachs, because the process is one of using observations to determine the specific nature of the geometry that exists out there by determining a set of unknown functions from astronomical observations, rather than fitting a few parameters to a pre-determined geometry.

This application has received great impetus in recent years because of the feature mentioned above: this determination of the Lemaitre-Tolman-Bondi (LTB) geometry $[3,12,18]$ from observations can be carried out for any value of the cosmological constant $\Lambda$, including $\Lambda=0$. This therefore provides a route to explain the apparent cosmic acceleration indicated by supernova observations without any dark energy: drop the assumption of spatial homogeneity and see if an LTB model fits the observations as well as the standard LCDM model, with no cosmological constant or dark energy present $[2,4]$. At first order, this is indeed possible (as follows from the theorem mentioned above): the supernova and number count observations can be accommodated in this way. Extensive investigations are now under way to see if all the other data (CMB observations, nucleosynthesis data, and so on) can also be fitted by these models. Because it goes into these kinds of issues in depth, this takes on aspects of a standard model-fitting exercise, as is usual in cosmology; for example multiple matter components and dark matter must be allowed for. It has been suggested by Moss et al. [16] that precision cosmology measurements already disprove this inhomogeneous model; but that conclusion is disputed inter alia by Biswas et al. [1] and Clarkson and Regis [5]. The issue relates to how to handle conditions on the central world line.

\section{Assessment}

This is a beautiful paper, carried out with precision and great understanding of issues involved, resulting from the interaction at between Ehlers, Schücking, and Sachs when they were in the Relativity Group of the University of Texas (created by Alfred Schild), stimulated and informed by lectures given by Gerard de Vaucouleurs of the Astronomy Department - who was an outstanding observer. Jerome Kristian was one of Sachs's students, along with others such as Ron Kantowski, Arthur Wolfe, and Beatrice Tinsley. The paper is also heavily influenced by the work of G C McVittie, who had carried out power series analyses of observational relations in FLRW models. The direct 
observational procedure proposed here has not received wide acclamation in the community of cosmology theorists, because of its lack of explanatory power relative to simple causal models: it determines what is there rather than explaining why it is there. However it is essentially the approach adopted by observationalists from Hubble on, as they sought to see what is actually there. Kristian and Sachs developed this approach into a systematic and complete relativistic scheme. Surveys such as the SSDS and $2 \mathrm{dF}$ in essence adopt this approach, albeit in a somewhat reduced way: their main analyses use only some of the data envisaged by Kristian and Sachs (in particular, they do not record proper motions, currently unobservable on cosmological scales), and many of their analyses are more model based than the Kristian and Sachs approach envisages (this is inevitable if one aims to bring into play the full range of data available today). However these surveys do record most of what would be needed to follow the Kristian and Sachs approach more directly in terms of reconstructing explicitly the geometry of the past light cone (see e.g. the discussion of SDSS data by York et al [19]), and doing so might be an interesting task. In any case these approaches let us see what is actually there - a very important dual to the usual more model-based fitting approaches. Each approach enlightens and informs the other.

The paper is also useful in helping clarify what is actually observable in cosmology, and what is not. We can expect its influence to last into the future.

\section{Jerome Kristian - a brief biography}

By A. Krasiński, abstracted from Ref. [20]

Jerome Kristian was born on 5th June 1934 in Milwaukee, Wisconsin.

He graduated from the University of Chicago, and there, in 1962, he obtained his PhD degree, supervised by S. Chandrasekhar.

In 1962-1964 he was an instructor at the University of Texas, where he collaborated with Rainer K. Sachs. The paper reprinted here is the result of that collaboration. After that, in 1966-1967, he worked at the University of Wisconsin as an Assistant Professor.

In 1968 he moved to the Mount Wilson Observatory, where he worked until his untimely death in 1996, first as a Carnegie Fellow, then as a full staff member.

Up until 1968, J. Kristian had been mainly a theoretician, but at the Observatory he changed his preferences and started working on observations. A large part of his work was concerned with the optical identification of X-ray and radio sources. He co-edited (with A. and M. Sandage) volume IX of the series Stars and Stellar Systems, entitled Galaxies and the Universe. He also collaborated with James A. Westphal on the use of silicon target detectors in the telescope. He participated in the discovery of super-massive objects in galactic nuclei.

For the last period of his life, J. Kristian worked with the Planetary Camera Team at the LST (later, Hubble Space Telescope). Not all of the results of that collaboration had been published prior to his death.

Jerome Kristian died on 22 June 1996 in an airplane accident. 
A brief biography of Rainer K. Sachs was printed together with another Golden Oldie in Gen. Relativ. Gravit. 39, 1941 (2007), doi:10.1007/s10714-007-0448-9.

\section{References}

1. Biswas, T., Notari, A., Valkenburg, W.: Testing the void against cosmological data: fitting CMB, BAO, SN and H0 (2010). arXiv:1007.3065v1

2. Bolejko, K., Krasiński, A., Célérier, M.N., Hellaby, C.: Structures in the Universe by exact methods: formation, evolution, interactions. Cambridge University Press, Cambridge (2009)

3. Bondi, H.: Spherically symmetrical models in general relativity. Mon. Not. R. Astron. Soc. 107, 410 (1947); reprinted, with historical comments, in Gen. Relativ. Gravit. 31, 1777 (1999)

4. Célérier, M.-N.: Do we really see a cosmological constant in the supernovae data?. Astron. Astrophys 353, 63-71 (2000). arXiv:astro-ph/9907206v4

5. Clarkson, C., Regis, M.: The cosmic microwave background in an inhomogeneous universe (2010). arXiv: $1007.3443 \mathrm{v} 1$

6. Ehlers, J.: Contributions to the relativistic mechanics of continuous media (in German), Akad Wiss und Lit (Mainz), Abh Mat-Nat Kl. Nr. 11, 792-837 (1961). English translation: Gen. Relativ. Gravit. 25, 1225-1266 (1993)

7. Ellis, G.F.R.: Relativistic cosmology. In: Sachs, R.K. (ed.) Proceedings of the International School of Physics "Enrico Fermi”, Course 47: General relativity and cosmology. Academic Press, pp. 104-182 (1971). Reprinted in Gen. Relativ. Gravit. 41, 581 (2009)

8. Ellis, G.F.R., Nel, S.D., Stoeger, W., Maartens, R., Whitman, A.P.: Ideal observational cosmology. Phys Reports 124, 315-417 (1985)

9. Etherington, I.M.H.: On the definition of distance in general relativity. Philosophical Magazine Nr. 18, 761 (1933). Reprinted in Gen. Relativ. Gravit. 39, 1055 (2007)

10. Hawking, S.W.: Perturbations of an expanding uniuverse. Astrophys. J. 145, 544 (1966)

11. Hellaby, C., Alfedeel, A.H.A.: Solving the observer metric. Phys. Rev. D79, 043501 (2009). arXiv:0811.1676

12. Lemaître, G.: L'Univers en expansion (The expanding Universe). Ann. Soc. Sci. Bruxelles A53, 51 (1933); English translation, with historical comments: Gen. Relativ. Gravit. 29, 637 (1997)

13. Lu, T.H.-C., Hellaby, C.: Obtaining the spacetime metric from cosmological observations. Class. Quantum Gravit. 24, 4107-4131 (2007). arXiv:0705.1060

14. McClure, M.L., Hellaby, C.: Determining the metric of the cosmos: stability, accuracy, and consistency. Phys. Rev. D78, 044005 (2008). arXiv:0709.0875

15. McCrea, W.H.: Observable relations in relativistic cosmology. II. Zeitschrift für Astrophysik 18, 98 (1939). Reprinted in Gen. Relativ. Gravit. 30, 312 (1998)

16. Moss, A., Zibin, J.P., Scott, D.: Precision cosmology defeats void models for acceleration. (2010). arXiv:1007.3725v1

17. Mustapha, N., Hellaby, C., Ellis, G.F.R.: Large scale inhomogeneity versus source evolution-can we distinguish them observationally?. Mon. Not. R. Astron. Soc. 292, 817-830 (1997). arXiv: gr-qc/9808079

18. Tolman, R.C.: Effect of inhomogeneity on cosmological models. Proc. Nat. Acad. Sci. USA 20, 169 (1934); reprinted, with historical comments: Gen. Relativ. Gravit. 29, 931 (1997)

19. York, D.G., et al. (The SDSS collaboration): The Sloan Digital Sky Survey: technical summary. Astron. J. 120, 1579-1587 (2000). arXiv:astro-ph/0006396

20. Munch, G.: Bull. Am. Astron. Soc. 29, 1476 (1997) 